\title{
Reflection Properties of Multimode Interference Devices
}

\author{
E. C. M. Pennings, Member, IEEE, R. van Roijen, M. J. N. van
}

Stralen, P. J. de Waard, R. G. M. P. Koumans and B. H. Verbeek

\begin{abstract}
We report on the reflection properties of multimode interference (MMI) devices: we distinguish between reflection back into the input waveguides and internal resonance modes due to the occurrence of simultaneous self-images. Because of self-imaging, reflection can be extremely efficient, even in the case of MMI devices with optimized transmission. This conclusion is confirmed by the observed spectral behavior of InP-based ring lasers incorporating MMI $3 \mathrm{~dB}$ couplers and MMI power splitters. Several techniques are proposed to minimize the influence of these reflections.
\end{abstract}

\section{INTRODUCTION}

$\mathbf{M}$ ULTIMODE interference (MMI) devices employing self-imaging [1], [2] in multimode waveguides have recently developed into key components for photonic integrated circuits. Several MMI devices have been reported, such as $3 \mathrm{~dB}$ couplers [3], [4], $90^{\circ}$ hybrids [5] and symmetric power splitters [6], [7]. Their unique properties, such as insensitivity to wavelength and polarization, good fabrication tolerance, low loss, compact size and stable splitting ratio, make MMI devices attractive components for integration in more advanced circuits, such as Mach-Zehnder modulators [8], [9] and an optical coherent front-end chip [10]. Recently, we have fabricated InP-based ring lasers employing MMI $3 \mathrm{~dB}$ couplers as the outcoupling elements and MMI power splitters to combine both counter-propagating beams [11]. The spectral behavior of these ring lasers shows effects that are to be associated with reflections in the MMI devices. So far, all analyses of MMI devices have focussed on transmission properties [1], [2], [4], [12]-[14]. In this paper, the reflection properties of MMI devices are analyzed in detail and we find that even devices with optimum transmission can show significant reflection. Several techniques are proposed to minimize this reflection.

\section{TRANSMISSION PROPERTIES OF MMI DEVICES}

Multimode interference (MMI) phenomena are a fundamental property of multimode waveguides and are due to a

Manuscript received January 27, 1994; revised March 29, 1994.

E. C. M. Pennings is with Philips Research Laboratories, Prof. Holstlaan 4, 5656 AA Eindhoven, The Netherlands.

R. van Roijen, P. J. de Waard, R. G. M. P. Koumans and B. H. Verbeek are with Philips Optoelectronics Centre, Prof. Holstlaan 4, 5656 AA Eindhoven, The Netherlands.

B. H. Verbeek is also with Delft University of Technology, Faculty of Electrical Engineering, Laboratory of Telecommunication and Remote Sensing Technology, PO Box 5031, 2600 GA Delft, The Netherlands.

M. J. N. van Stralen is with Delft University of Technology, Faculty of Electrical Engineering, Laboratory of Electromagnetic Research, PO Box 5031, 2600 GA Delft, The Netherlands.

IEEE Log Number 9401958 quadratic spacing of the propagation constants [4], [14], given by

$$
\beta_{\nu}-\beta_{0} \approx-\frac{\pi \nu(\nu+2)}{3 L_{\pi}}, \text { for modes } \nu=0,1,2,3, \cdots
$$

with

$$
L_{\pi} \approx \frac{4 n w_{\mathrm{eff}}^{2}}{3 \lambda_{0}}
$$

in the paraxial approximation. The MMI section is characterized by an effective width $w_{\text {eff }}$, a guide index $n$, a free-space wavelength $\lambda_{0}$ and the beat length $L_{\pi}$ between the fundamental mode $\nu=0$ and the first-order mode $\nu=1$. There are three different MMI phenomena [9]:

i) $\boldsymbol{N} \times \boldsymbol{N}$ general self-imaging [1], [2], [4], [14]: it follows from (1) that all modes interfere constructively at the selfimaging distance of $6 L_{\pi}$, irrespective of their excitation. A mirror image occurs at $3 L_{\pi}$ and an $m_{g}$-fold image is found at MMI lengths of $3 L_{\pi} / m_{g}$. General self-imaging allows the use of arbitrary forms of excitation, such as bends, multimode access guides and reflecting corner mirrors, and can improve fabrication tolerances by increasing the pitch between the access guides.

ii) $2 \times \boldsymbol{N}$ restricted self-imaging [4], [14] occurs when $\nu=0,1,3,4,6,7, \cdots$ modes are excited only. This can be achieved by locating the input guides at $w / 3$ and at $2 w / 3$, i.e. the common zeros of modes $\nu=2,5,8, \cdots$. The self-imaging distance is $2 L_{\pi}$, a mirror image occurs at $L_{\pi}$ and an $m_{r^{-}}$ fold image is found at lengths $L_{\pi} / m_{r}$. The requirement with respect to the location of the access guides, however, leads to wider MMI sections and cancels a potential length reduction.

iii) $1 \times N$ symmetric power splitting [6,7] occurs when the MMI section is fed by a single central waveguide. Symmetric modes $\nu=0,2,4, \cdots$ are excited only and a self-imaging distance of $3 L_{\pi} / 4$ is found. The $m_{s}$-fold image is found at a length of $3 L_{\pi} / 4 m_{s}$ and with a pitch between the output guides of $w / m_{s}$.

\section{Reflection Properties of MMI Devices}

In order to be able to study reflection in MMI devices, we have used modal analysis-based propagation modelling [14] in conjunction with the effective index method: after the propagation constants of all guided modes and their respective lateral field distributions have been solved, device performance is simulated through repeated application of propagator matrices to account for phase evolution and matrices consisting of 
TABLE I

Transmission and Reflection Modes Inside MMI 3 dB Couplers $(L=200 \mu \mathrm{m})$ and MMI Power SpltTters $(L=64 \mu \mathrm{m})$. Waveguide Parameters are Described in the TeXt. Amplitudes are for Loss-Less MMI Devices and are Relative to the Field Reflection Coefficient $r$ at the End of the MMi Section. Phases are Relative to Each Other at One Plane and for Identical Directions of Propagation.

\begin{tabular}{|c|c|c|c|c|}
\hline Device & MMI 3dB Coupler & \multicolumn{3}{|c|}{ MMI Power-Splitter } \\
\hline Excitation & Single Input & Single Input & In-Phase Inputs & Out-of-Phase Inputs \\
\hline Transmission & $\infty^{i \sqrt{0.5}}$ & $\sqrt{0.5}$ & $\begin{array}{l}\sqrt{0.5} \\
-\infty\end{array}$ & $\begin{array}{l}-\frac{\sqrt{0.5}}{-28=80} \\
-\frac{-\sqrt{3}=80}{-\sqrt{0.5}}\end{array}$ \\
\hline $\begin{array}{l}\text { Back - } \\
\text { Reflection }\end{array}$ & & 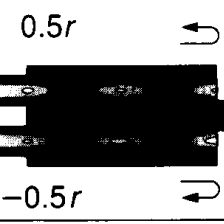 & & 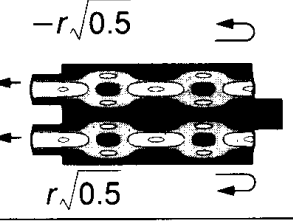 \\
\hline $\begin{array}{l}\text { Internal } \\
\text { Resonance }\end{array}$ & & & - & \\
\hline
\end{tabular}

overlap integrals to account for coupling at the junctions. This technique is, by its nature, very suitable for studying MMI phenomena and provides additional insight into the modal interference mechanism. Reflection calculation starts with the coupling between the exciting field in the input guides and the MMI section. Subsequent application of the propagator and coupling matrices yields the field distributions at each side of the junction of the MMI section and the output guides. The non-negligible difference between these two field distributions is split into a fraction $1-|r|^{2}$, which accounts for transmitted radiation, and a fraction $|r|^{2}$, which accounts for total reflection, where we have assumed that the guide-cladding interface at the junction of the MMI section and the output guides may be represented in terms of a single field reflection coefficient $r$. The value of $r$ may be small for weakly guiding (buried) waveguide structures, but can be quite large for deeply etched waveguides, especially when they are covered by a contacting metal layer. Reflection back into each input guide is finally calculated by propagating the total reflection at the end of the MMI section backwards through application of the coupling, propagator and coupling matrices respectively. Simulations have been performed for the waveguide parameters of the ring lasers, which are described further on. The results of these simulations, which are summarized in Table 1, may be divided into two different reflection mechanisms:

i) internal resonance modes are caused by the presence of several simultaneously occurring self-images. For example, the MMI $3 \mathrm{~dB}$ coupler is based on the two-fold $m_{g}=2$ image occurring at a length of $3 L_{\pi} / m_{g}=3 L_{\pi} / 2$. This length equals precisely twice the self-imaging length $3 L_{\pi} / 4$ for symmetric excitation $\left(m_{s}=1\right)$. Self-imaging ensures perfect imaging of both reflecting ends onto each other, thereby maximizing reflection and leading to an "internal resonance mode". The MMI power splitter, on the other hand, uses the two-fold image $m_{s}=2$ of the symmetric self-imaging, which occurs at a length of $3 L_{\pi} / 4 m_{s}=3 L_{\pi} / 8$. An "internal resonance mode" exists as well and consists of a mirrored version of the transmitted image (also $m_{s}=2$ ) as shown in Table I: self-imaging gives maximum intensity at the reflecting end of the MMI section. The transmission state and the internal resonance state thus occur for the same optimum length of the MMI section and these states are maximally decoupled at this length. For non-optimum lengths of the MMI section, self-images are imperfect and coupling between the two selfimages leads to reflections back into the access guides. The calculations shown in Fig. 1 for the MMI 3dB-coupler show that the (power) reflection may be smaller than $10^{-4} \cdot|r|^{2}$ for the optimum length, but can be as large as $\approx 0.5|r|^{2}$ for lengths deviating from the optimum length by $\pm 20 \%$.

ii) reflection back into the access guides: when the MMI power splitter is used in reverse as a power combiner, efficient combining operation requires inputs of equal amplitude and phase. If, however, the two inputs are $180^{\circ}$ out of phase, power is minimum in the output guide but maximum at the reflecting end of the MMI section. This leads to a perfect imaging of the input guide back onto itself, which is so efficient that the back 


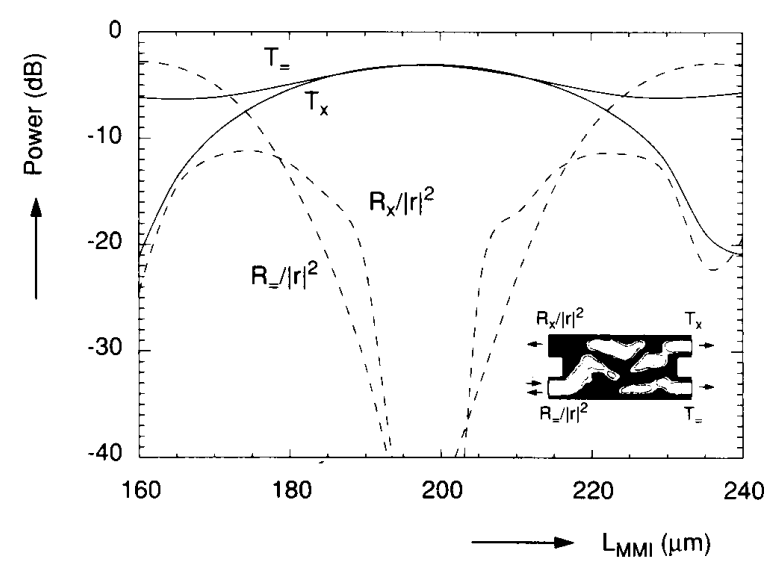

Fig. 1. Transmitted $\left(T_{=}\right.$and $\left.T_{x}\right)$ and reflected $\left(R_{=}=\left.|| r\right|^{2}\right.$ and $\left.R_{x} /|r|^{2}\right)$ power for MMI $3 \mathrm{~dB}$ couplers (for bar and cross guides, respectively). Waveguide parameters are described in the text and lead to an optimum length of $198 \mu \mathrm{m}$. Amplitudes are for loss-less MMI devices and reflected power is relative to the reflection $|r|^{2}$ at the end of the MMI section.

reflection into the input guides equals the reflection at the end of the MMI section $|r|^{2}$. Back reflection can thus vary from a minimum for in-phase excitation to a maximum for out-ofphase excitation for a single device optimized for maximum transmission.

\section{REFLECTIONS OBSERVED IN RING LASERS}

The presence of reflections in MMI devices, discussed above, has been observed in the spectral behavior of InP-based ring-lasers incorporating MMI 3dB couplers and MMI power splitters, as reported elsewhere [11]. Two different, completely active, ring laser configurations were fabricated in an MOVPEgrown double heterostructure with a $0.15 \mu \mathrm{m}$ thick active GaInAsP layer $\left(\lambda_{g}=1.6 \mu \mathrm{m}\right)$. Waveguides $(w=2.5 \mu \mathrm{m})$ were created by etching completely through the active layer using $\mathrm{Cl}_{2}$-based RIE. Five different MMI $3 \mathrm{~dB}$ couplers $(w=7 \mu \mathrm{m}$, $L=185-233 \mu \mathrm{m}$, access guide pitch $=4.5 \mu \mathrm{m})$ were used as outcouplers and five different MMI power splitters $(w=8 \mu \mathrm{m}$, $L=61-75 \mu \mathrm{m}$, access guide pitch $=4 \mu \mathrm{m}$ ) were used to combine both counter-propagating beams in a single output guide. Silicon-nitride passivation and a standard p-contacting layer were used. Lasers were mounted epi-side down for optimum heat conduction. After cleaving, lasers were AR coated using a single $\mathrm{HfO}_{2}$ layer $\left(n \approx 1.91, d \approx 0.27 \lambda_{0}\right)$ leading to a calculated residual reflection of $|r|^{2} \approx 0.6 \%$.

The presence of reflections in the MMI $3 \mathrm{~dB}$ coupler can be observed in the particular laser spectrum shown in Fig. 2; the left-hand side is dominated by a single lasing mode and reveals a mode spacing which corresponds to the circumference of the ring, whereas the right-hand side shows a Fabry-Perot type of spectrum with a very different mode spacing corresponding to the length of the MMI $3 \mathrm{~dB}$ coupler. Apparently, the MMI $3 \mathrm{~dB}$ coupler simultaneously operates in a transmission state (ring laser mode) and in an internal resonance state (MMI modes) (compare Table I and Fig. 2). Decoupling between these two states occurs for optimum lengths of the MMI section as shown

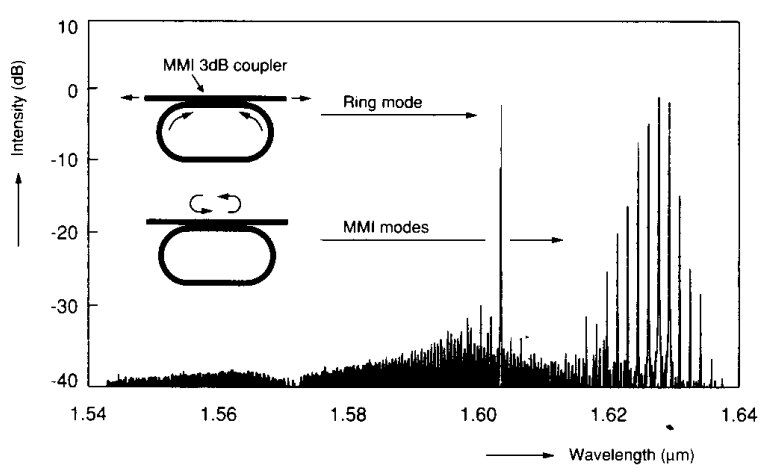

Fig. 2. Spectrum of ring laser with MMI $3 \mathrm{~dB}$ outcoupler at $2.1 \mathrm{I}_{\mathrm{th}}(412 \mathrm{~mA})$. The insets show schematic lay-outs and the two different lasing modes.

in Fig. 1. Their spectral separation, as shown in Fig. 2, has been observed at large currents only. Note that the MMI modes in the spectrum have a well-defined mode spacing and that they resemble the spectrum of a Fabry-Perot cavity of a single mode waveguide, whereas there are six laterally guided modes in the MMI section. The absence of mode competition between these six modes is explained by their constructive interference due to self-imaging.

The influence of reflections in the MMI combiner has been observed in the spectral behavior of the combiner laser (see Fig. 3). Extremely stable single-mode behavior with sidemode suppression in excess of $35 \mathrm{~dB}$ and occasional modehopping have been found, which is typical of coupled cavity behavior[15] and indicates coupling between the central ring and a second cavity. Fourier analysis of several spectra shows that the second cavity must have been formed by the MMI $3 \mathrm{~dB}$ coupler and the two outer semicircles all the way up to and including the MMI combiner (see the inset in Fig. 3). The two operating modes for the MMI combiner (see Table I) allow for two distinct lasing modes: i) a symmetric mode with in-phase excitation of the MMI combiner, efficient combining and phase locking through reflection from the cleaved output facet and ii) an asymmetric mode with out-of-phase excitation of the MMI combiner, inefficient combining and phase locking through reflection from the combiner. The Fourier analysis of the spectrum and the observed two-lobed near-field pattern of the laser output, demonstrate that the combiner laser has indeed been stabilized by the unintended reflection from the MMI combiner, which, consequently, must be larger than the amplified reflection from the AR-coated output facet.

Please note the functional similarity between the ring laser with MMI combiner and a laser with an external modulator of the Mach-Zehnder type. This similarity suggests a strong reflection mechanism back into the laser during the off-state of the Mach-Zehnder modulator.

\section{CONCLUSIONS}

In this paper, two different reflection mechanisms have been found for MMI devices: an internal resonance state due to different coexisting self-images and back reflection that depends on the relative phases of the inputs. Because 


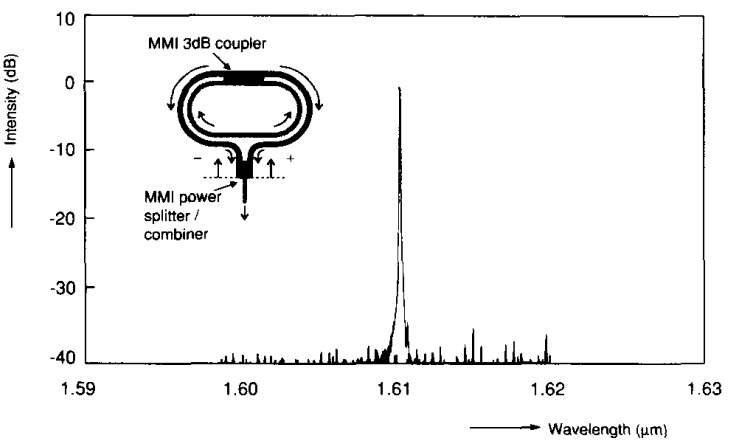

Fig. 3. Spectrum of ring laser with MMI $3 \mathrm{~dB}$ outcoupler and MMI combiner at $1.6 \mathrm{I}_{\mathrm{th}}(444 \mathrm{~mA})$. The inset shows the schematic lay-out and the asymmetric lasing mode with reflection from the combiner.

of self-imaging, reflection back into the input guides can be extremely efficient, even in the case of devices with optimized transmission, and can be as large as the reflection coefficient $|r|^{2}$ at the end of the MMI section. This conclusion is in very good agreement with the observed spectral behavior of ring lasers incorporating MMI devices. The experiments show that reflections caused by MMI devices can have great effects on the properties of reflection-sensitive photonic integrated circuits, such as lasers or coherent receivers and this, in turn, indicates a need to control the influence of these reflections. Lasing action inside the MMI section may be prevented by employing passive waveguide material for the MMI section. The effectiveness of reflections at the end of the MMI section can be reduced by using angled or rough ends, by applying AR coatings, or by using weakly guiding waveguides. An alternative approach is to adapt the MMI section at those sites where the reflected intensity is great and where the transmitted intensity is small, for example by means of absorbers or tapering. A tapered MMI power splitter (see Table I) reduces both types of reflections and may in addition relax the requirements of the photolithographic definition. Simulations show that transmission efficiency is not affected when the MMI- section is tapered according to the full angle beam divergence given by $2 \theta \approx 4 \lambda_{0} /(\pi n w) \approx 18^{\circ}$, where $w \approx 2 \mu m$ is the $e^{-2}$ field diameter in the output guide. We expect that these findings may improve the performance of multimode interference devices and may lead to new applications.

\section{ACKNOWLEDGMENT}

The authors are most grateful to T. van Dongen and J.J.H. van der Heijden for the preparation of the ring lasers.

\section{REFERENCES}

[1] O. Bryngdahl, J. Opt. Soc. Am., vol. 63 no. 4, pp. 416-419, 1973.

[2] R. Ulrich and G. Ankele, Appl. Phys. Lett., vol. 27, no. 6, pp. 337-339, 1975

[3] E. C. M. Pennings, R. J. Deri, A. Scherer, R. Bhat, T. R. Hayes, N. C. Andreadakis, M. K. Smit, L. B. Soldano and R. J. Hawkins, Appl. Phys. Lett., vol. 59, no. 16, pp. 1926-1928, 1991.

[4] L. B. Soldano, F. B. Veerman, M. K. Smit, B. H. Verbeek, A. H. Dubost, E. C. M. Pennings, J. Lightwave Technol., vol. 10, no. 12, pp. 1843-1850, 1992.

[5] E. C. M. Pennings, R. J. Deri, R. Bhat, T. R. Hayes and N. C Andreadakis, IEEE Photon. Technol. Lett., vol. 5, no. 6, pp. $701-703$ 1993.

[6] L. B. Soldano, M. Bouda, M. K. Smit and B. H. Verbeek, Proc. 18th ECOC '92, Paper WeB10, pp. 465-468.

[7] J. M. Heaton, R. M. Jenkins, D. R. Wight, J. T. Parker, J. C. H. Birbeck and K. P. Hilton, Appl. Phys. Lett., vol. 61, no. 15, pp. 1754-1756, 1992

[8] J. E. Zucker, K. L. Jones, T. H. Chiu, B. Tell, and K. Brown-Goebeler, J. Lightwave Technol., vol. 10, no. 12, pp. 1926-1930, 1992.

[9] M. Bachmann, M. K. Smit, P. A. Besse, E. Gini, H. Melchior, L. B Soldano, Proc. OFC/IOOC' 93 , Paper TuH3, pp. 32-33.

[10] R. J. Deri, E. C. M. Pennings, A. Scherer, A. S. Gozdz, C. Caneau, N. C Andreadakis, V. Shah, L. Curtis, R. J. Hawkins, J. B. D. Soole and J.-I. Song, IEEE Photon. Technol. Lett., vol. 4, no. 11, pp. 1238-1240, 1992.

[11] R. van Roijen, E. C. M. Pennings, M. J. N. van Stralen, J. M. M. van der Heijden, T. van Dongen and B. H. Verbeek, Appl. Phys. Lett., vol 64, no. 14, pp. 1753-1755, 1994.

[12] D. C. Chang and E. F. Kuester, IEEE Trans. Microw. Theory Techn., vol. MTT-29, no. 9, pp. 923-933, 1991.

[13] P. A. Besse, M. Bachmann and H. Melchior, Proc. ECIO, 1993, pp 2-22 and 2-23.

[14] E. C. M. Pennings, "Bends in optical ridge waveguides: modeling and experiments", Ph.D.Thesis, 1990, Delft University of Technology, The Netherlands, ISBN 90-9003413-7.

[15] G. P. Agrawal and N. K. Dutta, "Long-wavelength semiconductor lasers", (Van Nostrand Reinhold Company, New York, 1986). 